\title{
Future advances in robotic dentistry
}

\begin{abstract}
Robotics is a next generation technology which opened new pathways for it to expand and explore the various areas of dentistry and it also helps in fulfilling tasks which is difficult for the dental clinician to achieve. Dental robots have various uses like teaching dental students, endo micro robot, arch wire bending and dental Implantology. This focused review aims at application of robotics in various specializations in dentistry.
\end{abstract}

Volume 7 Issue 3 - 2017

\section{Pradeep Kumar Y, Prashansa Dixit, Kalaivani V, Rajapandian $\mathrm{K}$ \\ Department of Periodontics, SRM University, India}

\author{
Correspondence: Pradeep Kumar, Department of \\ Periodontics, SRM Kattankulathur Dental College, SRM \\ University, Chennai-603 203,Tamil Nadu, India, \\ Email yadalam1980@gmail.com
}

Received: April 15, 2017 | Published: May 18, 2017

\section{Introduction}

Robotics is that branch of technology which specializes in designing, construction, operation and application of robots as well as the computer systems for its control, sensory feedback and processing the information. It was introduced by writer Isaac Asimov in his science fiction book named I robot which was published in 1950. According to the robot institute of America a robot is defined as "a reprogrammable , multifunctional manipulator designed to move materials, parts, tools or specialized devices through various programmed motions for the performance of a variety of tasks". ${ }^{1}$

\section{Robots in dental field}

Dental patient robots: The concept of dental patient robot currently called as phantom heads, which consist of simple functional cephalic region and arrangement of teeth were found out to be much different from actual patients. This concept of dental patient robots was initiated in Japan.

Show hanako: In the capital of Japan, Showa University mingled with the robotics company named TMSK in order to manufacture a realistic robot which behaved exactly like a human in its gestures and responses which helped the dental students to learn in a better way. ${ }^{2}$

Geminoid DK: It is the first robot in a series based on personalities outside Japan. These robots are called geminoids and are remote controlled with advanced motion capture technology allowing the machine to copy the facial expressions and the various head motions. ${ }^{3}$

Simroid: This robot was developed at The Nippon Dental University Kokoro and is used to train dentists. It is an upgrade to simuloid a less efficient robot which was developed in 2007. This robot is developed in order to provide an emotional feedback to dentists especially pain and discomfort, also responds and reacts to questions and commands and finally rating and evaluating their treatment. ${ }^{4}$

\section{Endo micro robot}

It was developed to reduce human error and increase the quality of endodontic treatment. It aims at developing a technique which assesses the tooth conditioning using the art of computer graphics and tooth models, computer aided procedure planning, designing and building a smart multipurpose micro machine to perform machine controlled root canal treatment and finally an ultrasonic cleaning device which helps in waste removal. ${ }^{5,6}$ This endo-micro robot basically consists of a micro position and orientation adjustment device, an automatic feed rate and travel distance controller, micro sensors and apex sensors with flexible drills and vacuum attachments. ${ }^{7}$

\section{Surgical robots}

The intervention of robotics into surgery has allowed surgeons to create a new kind of environment in the operating room. Oral and maxillofacial surgery has a new kind of surgical robot which is programmed beforehand in order to perform the various functions in the operation theatre and also interact with the surgeon. ${ }^{8-10}$

\section{Sensor equipped implant setup}

Recently this new system of computer assisted surgery for implants has been developed consisting of pre operative and intra operative stage. In the preoperative stage it uses the $3 \mathrm{D}$ views obtained from the raw images of the patient before surgery followed by the intra operative stage in which it shows the 3D orientation of surgical instrument position and trajectories which are displayed on the monitor within a patient's 3D imaging data. A vigorous and highly precise tracking system is the main parameter in navigation system. In order to check the position of the patient and the movement of the surgical instruments an infrared based surgical marker emitter is designed in various patterns..$^{11,12}$

\section{Robotic dental drill}

It is a new advancement which is developed by tactile technologies and consists of immobilizing the jaw of the patient and suspending thin needles which will penetrate the gum and determine the location of the bone. This whole unit is connected with a wireless connection to a PC and joins with the CT scan data thereby producing a set of drill guides which once activated is self directing and can be altered by the clinician as per the requirement. ${ }^{13}$

\section{Tooth arrangement robot}

It is a single manipulator system which is used for the manufacturing of complete dentures. It was developed using 6 degree of freedom CRS robot and produced in Canada. ${ }^{14-16}$ The various functions of this software are to choose and create medical history files of the patient 
followed by drawing a jaw arch and dental arch curves and finally according to the jaw arch parameters adjust the dental arch curve. It also displays the $3 \mathrm{D}$ virtual dentitions on the screen with a careful observational environment and aids in modification of the individual tooth posture. Its functions are to adjust the tooth arrangement initial position for the robot, create control data profile and control the robot for tooth arrangement operations. This robot system was used in the manufacture of upper and lower complete dentures. This scheme of tooth arrangement multifinger hand is designed based on MOTOMAN UP6 robot. These hands consist of three fingers for working in the three degrees of freedom and thus are able to achieve the tooth arrangement necessities theoretically. There are around 14 individual manipulators on the dental arch curve. This manipulator assist every tooth by tooth arrangement helper and supplies the controls with six degrees of freedom to modify each tooth for its position along X, Y, Z, lingual, rotation and near-far medium directions. The manufacturing of complete dentures takes only 30 minutes using this robot system and the precession and accuracy of each robotic system is measured. ${ }^{17}$

\section{Dental implantology robot}

This system mimics the mandibular movements and occlusal contact forces in order to make it possible for various implant designs and procedures to be tested and evaluated before animal testing or clinical human trials. This technology of robot implant denture from preoperative planning to splint drilling was established by Ecole des Mines de Paris in France, and Umea Universities in Sweden. This method consists of forming pre programmed software which is used to work with CT scanner data. Now a 3D model of the patients jaw is made, implant fixtures are placed and an accurate robot is then used to drill a jaw splint in the predetermined locations in order to make a surgical guide. This robot has three translations and two rotations. ${ }^{18,19}$ Another system using the ABB IRB2400/M98 robot is brought about by University of Coimbra in Portugal. This system is well equipped and includes industrial robot manipulator, a data accqusition board, strain gauges and a torque sensor. It performs the implant drilling and applies pressure on the gathered implants to mimic mastication process. ${ }^{20}$ An implant surgery system built by the University of Duesseldorf consists of a robot arm, angle sensors, torque sensors, an accuracy potentiometer, driver shafts and mini implants in bone. It is primarily concerned with the effect of insertion angle and depth and stability and predrilling diameter of the mini implant..$^{21,22}$

\section{Orthodontic arch wire bending robots}

This technology for automatically bending orthodontic wires was devices by Werner Butscher. The bending apparatus is known as Suresmile arch wire bending robot. ${ }^{23}$ It is an all new imaging system which uses 3D imaging and computer techniques for diagnosis and treatment planning and also the application of CAD CAM improves efficiency and quality of work..$^{24,25}$ The various devices like lingual arch wire manufacturing and design aid (LAMDA) have been established by Gilbert. It has movement only in XY plane. ${ }^{26}$ Another type of arch wire bending robot is based on MOTOMAN UP6 is composed of PC and arch wire bending actuator. This connects with the MOTOMAN robot end and is used to stabilize and bend the arch wire. ${ }^{27,28}$ The various other things analyzed by this robot include bending position, angle of optimization of the arch wire, the kinematics and bending properties. ${ }^{29-35}$ Cartesian type is another type of arch wire bending robot which consists of base, rotary, feed and supporting structure of arch wire bending die and bending mechanism. ${ }^{36,37}$

\section{Conclusion}

The intervention of robotics in the field of dentistry can offer improved and precise treatment with good quality of work in a less amount of time. It can alter the dental health of the people and will be much safer. With the emerging new technologies the future of dentistry is uncertain and our matter of concern lies in the fact that the technology should be acceptable by the people and we as clinicians are able to use this technology in our daily teaching and clinical practice.

\section{Acknowledgments}

None.

\section{Conflicts of interest}

The author declares that there is no conflict of interest.

\section{References}

1. https://en.oxforddictionaries.com/definition/robotics

2. http://geminoid.dk.sci-hub.ac/

3. http://gizmodo.com/5862512/simroid-trains-future-human-slaves-inrobot-dental-hygiene

4. http://www.diginfo.tv.sci-hub.ac/v/11-0245-r-en.php

5. West JD, Roane JB. Cleaning and Shaping the Root Canal System. In Cohen S, et al. (Eds.), Pathways of the Pulp. (7th edn), C.V. Mosby, Missouri, USA, 1997. p. 203-257.

6. Dong J. Rule-based Planning for Automated Endodontic TreatmentFrom Dental Radiography, 3-D Computer Modeling, to Tool Selection and Path Control. Dissertation, Columbia University. 2003. p. 149-153.

7. Schulz MJ, Shao VN, Yun Y. Nanomedicine Design of Particles, Sensors, Motors, Implants, Robots, and Devices, Artech House, London, UK, 2009. p. 1-10.

8. DiGioia AM, Colgan BD, Koerbel N. Computer aided surgery. In: Satava RM, editor. Cybersurgery: Advanced Technologies for Surgical Practice. John Wiley \& Sons, New York, USA, 1998. p. 121-139.

9. Lueth TC, Hein A, Albrecht J, et al. A Surgical Robot System for Maxillofacial Surgery. IEEE, Germany, Europe, 1998. p. 2470-2475.

10. Ing WS “3D Planning System for Dental Implantology.

11. Hoffmann WJ, Roman GG, Reinert S. Accuracy of navigation-guided socket drilling before implant installation compared to the conventional free-hand method in a synthetic edentulous lower jaw model. Clin Oral Implants. 2005;16(5):609-614.

12. Edinger DH. Planning and implant positioning by a robot system in the dental practice. Digital Dental News. 2012;6:32-38.

13. http://technews.tmenet.com/robotxworld/topics/robotics/ articles/130220-\%20cerec-ac-robot-introduced-china-shesays.htm

14. Zhang YD, Zhao ZF, Lu JL, et al. "Robotic manufacturing of complete dentures". IEEE, USA, 2001. p. 2261-2266.

15. Song RJ, Zhang YD, Zhao ZF, et al. A Tooth arrangement algorithm for robot aided denture processing. Journal of Beijing Institute of Technology. 2001;21(4):474-479.

16. Zhang YD, Zhao ZF, Song RJ, et al. Tooth arrangement for the manufacture of a complete denture using a robot. Industrial Robotvol. 2001;28(5):420-425. 
17. Zhang Y, Ma J, Zhao Y, et al. Kinematic analysis of tooth-arrangemen robot with serial-parallel joints. IEEE, Hunan, China, 2008. p. 624-628.

18. Dutreuil J, Goulette F, Laurgeau C, et al. Computer assisted dental implantology: a new method and a clinical validation. Medical Image Computing and Computer-Assisted Intervention-MICCAI. 2001. p. 384 391.

19. Chen XJ, Wang CT, Lin YP. A computer-aided oral implantology system. Proceedings of theIEEE-EMBS 27th Annual International Conference of the Engineering in Medicine and Biology Society. 2005. p. 3312-3315.

20. Pires JN, Caramelo FJ, Brito P, et al. Robotics in implant dentistry: stress/ strain analysis. System overview and experiments. Industrial Robot. 2006;33(5):373-380.

21. Wilmes B, Su YY, Drescher D. Insertion angle impact on primary stability of orthodontic mini-implants. Angle Orthodontist. 2008;78(6):10651070 .

22. Wilmes B, Drescher D. Impact of insertion depth and predrilling diameter on primary stability of orthodontic mini-implants. Angle Orthodontist. 2009;79(4):609-614.

23. Rigelsford J. Robotic bending of orthodontic archwires. Industrial Robot. 2004;31(6):321-335.

24. Muller Hartwich R, Präger TM, Jost-Brinkmann PG. SureSmile$\mathrm{CAD} / \mathrm{CAM}$ system for orthodontic treatment planning, simulation and fabrication of customized archwires. International Journal of Computerized Dentistry. 2007;10(1):53-62.

25. Alford TJ, Roberts WE, Hartsfield JK, et al. Clinical outcomes for patients finished with the SureSmile method compared with conventional fixed orthodontic therapy. Angle Orthod. 2011;81(3):383-388.

26. Gilbert A. An in-office wire-bending robot for lingual orthodontics," Journal of Clinical Orthodontics. 2011;45(4):230-236.

27. Zhang Y, Jia Y. The control of archwire bending robot based on MOTOMAN UP6. Proceedings of the 2nd International Conference on Biomedical Engineering and Informatics, China, 2009. p. 1-5.
28. Du H, Jia Y, Zhang Y, Liu Y. Trajectory planning of archwire bending robot. China Mechanical Engineering. 2010;21(13):1605-1608.

29. Jiang JX, Zhang YD, Liu YJ. Algorithms comparison of control points planning of target archwire. ICIC Express Letters. Part B: Applications. 2012;3(4):923-930.

30. Zhang YD, Jiang JX. Trajectory planning of robotic orthodontic wires bending based on finite point extension method. Advanced Materials Research 201-203. 2011. p. 1873-1877.

31. Jiang JX, Zhang YD. Searching control points of formed archwire based on dichotomy and multi-objective optimization method. Journal of Computational Information Systems. 2012;8(6):2309-2316.

32. Zhang Y, Jiang J. Optimization algorithm of control points planning of archwire bending forming. International Journal of Digital Content Technology and its Applications. 2012;6(22):590-599.

33. Zhang Y, Jiang J. Bending property analysis and experimental study of orthodontic wires. China Mechanical Engineering. 2011;22(15):18271831

34. Zhang Y, Jiang J. Kinematics analysis and simulation study of an archwire bending robot. Chinese High Technology Letters. 2012;22(7):727-734.

35. Zhang Y, Jiang J. Analysis and experimentation of the robotic system for archwire bending. Applied Mechanics and Materials. 2012(121)126:3805-3809.

36. Jiang JG, Zhang YD, Zhou K, et al. Structure design of archwire bending robot for orthodontic treatment. Proceedings of the 8th International Forum on Strategic Technology, Ulaanbaatar, Mongolia, 2012. p. 696699.

37. Jingang $\mathrm{J}$, Yongde $\mathrm{Z}$, Mingliang $\mathrm{J}$, et al. Bending process analysis and structure design of orthodontic archwire bending robot. International Journal of Smart Home. 2013;7(5):345-352. 